Z. Klin. Chem. Klin. Biochem.

11. Jg. 1973, S. $535-536$

\title{
Enzymatische Endwertmethode zur Xylit-Bestimmung
}

\author{
Von C. Maurer und P. Christophis \\ Aus dem Klinisch-Chemischen Laboratorium \\ (Leiter: Priv. Doz. Dr. C. Maurer) der Chirurgischen Klinik der Universität Heidelberg
}

(Eingegangen am 29. August/8. Oktober 1973)

Es wird ein enzymatischer Xylit-Nachweis mit Sorbit-Dehydrogenase beschrieben. Das Enzym katalysiert eine Gleichgewichtseinstellung, die auf der Seite von Xylit liegt. Durch Zusatz von Hydrazinsulfat ist es möglich, die gebildete D-Xylulose als Hydrazon abzufangen und einen quantitativen Umsatz von Xylit zu erzielen. Auch höhere Fructosc-Konzentrationen, die wegen der fehlenden Spezifität des Enzyms mit dem gebildeten $\mathrm{NADH}$ unter Rückverwandlung zu NAD+ reagieren, können durch den Hydrazin-Zusatz aus dem Bestimmungsansatz entfernt werden.

Der Nachweis ist methodisch einfach und eignet sich deshalb für die routinemäßige Bilanzierung bei parenteraler Zufuhr von Xylit.

\section{Enzymatic endpoint determination of xylutol}

An enzymatic determination of xylitol by sorbitol-dehydrogenase is described. The equilibrium catalyzed by the enzyme favours the formation of xylitol. By adding hydrazine sulfate it is possible to trap D-xylulose as its hydrazone and to acquire a quantitative turnover of xylitol. Fructosc, which interferes becausc of the lack of specifity of the enzyme, is eliminated, cven in high concentrations, by addition of hydrazine. The determination is methodically facile and therefore suitable for routine balance determinations, following the parenteral application of xylitol.

In der parenteralen Ernährung findet Xylit neben Glucose und Fructose als "Ersatz-Kohlenhydrat" zunehmend Verwendung. Für die Bilanzierung der hochkalorischen Ernährung in der postoperativen Behandlung und bei der "Hyperalimentation" bei schweren Magen-Darm-Erkrankungen ist eine sichere Erfassung der Xylit-Ausscheidung und eine Abgrenzung gegenüber Glucosurie und Fructosurie wichtig. Der Xylit-Nachweis mit NAD+ und Sorbitdehydrogenase (Polyol-Dehydrogenase, EC 1.1.1.14) im enzymatischkinetischen Test (1) setzt konstante Meßbedingungen (Temperatur, pH-Wert, neue Eichkurve für jede Enzymcharge) voraus, die im klinischen Routinelaboratorium nur schwer einzuhalten sind. Höhere Fructosekonzentrationen interferieren durch Oxidation des gebildeten $\mathrm{NADH}$ wegen der mangelnden Spezifität des Enzyms.

\section{Methodik}

\section{Prinzip}

Xylit $+\mathrm{NAD}^{+} \rightleftharpoons \mathrm{D}$-Xylulosehydrazon + $\mathrm{NADH}+\mathrm{H}^{+}$

Die NADH-Zunahme gemessen bei 340 (334 oder 366) $\mathrm{nm}$ ist Meßgröße. Fructose wird als Fructose-hydrazon aus dem Bestimmungsansatz entfernt.

\section{Optimale Meßbedingungen}

Das Gleichgewicht der Reaktion wird im alkalischen Milieu zugunsten der Dehydrierung von Xylit verschoben. Das pH-Optimum des Enzyms liegt zwischen pH 8,0 und 8,6. Im stärker. alkalischen Milieu läuft daher die Reaktion mit wesentlich geringerer Geschwindigkeit ab.

\section{Reagenzien}

1. Tris-(hydroxymethyl)-aminomethan

2. Hydrazinsulfat

3. $\beta$-Nicotinamid-adenin-dinucleotid $\left(\beta-\mathrm{NAD}^{+}\right)$. (Boehringer

Mannheim)

4. Sorbitdehydrogenase (L-Iditol: NAD oxidoreductase, EC 1.1.1.14) (Boehringer Mannheim; Calbiochem; Koch, Ligth \& Co.) 5. Xylit (Merck, Darmstadt)

6. Perchlorsäure.

\section{Herstellung der Lösungen}

Alle Lösungen müssen mit doppelt destillierten oder demineralisierten Wasser hergestellt werden.

1. Tris $1 \mathrm{~mol} / \mathrm{l} \mathrm{mit}$ Hydrazinsulfat $0,4 \mathrm{~mol} / \mathrm{l}$ :

$12,1 \mathrm{~g}$ Tris p. a. im 100-ml-Meßkolben lösen. 5,2 g Hydrazinsulfat hinzufügen. Mit $1 \mathrm{~mol} / 1 \mathrm{HCl} \mathrm{pH} 8,5$ einstellen und bis zur Marke auffüllen.

2. $\beta$-Nicotinamid-adenin-dinucleotid $\left(\beta-\mathrm{NAD}^{+}\right) 60 \mathrm{mmol} / \mathrm{l}$ :

$400 \mathrm{mg}$ NAD werden in $10 \mathrm{ml}$ Wasser gelöst.

3. Sorbitdehydrogenase $1 \mathrm{mg} / \mathrm{ml}$ in Wasser gelöst.

4. Xylit-Kontroll-Standard $500 \mathrm{mg} / \mathrm{l}$ :

$50 \mathrm{mg}$ Xylit p. a., getrocknet, werden in $100 \mathrm{ml}$ Wasser gelöst.

5. Perchlorsäure $0,6 \mathrm{~mol} / \mathrm{l}$ :

Perchlorsäure konzentriert p. a. $600 \mathrm{~g} / \mathrm{kg}$ im Verhältnis $1: 15$ verdünnen.

\section{Probennahme und Vorbereitung}

Vollblut wird nach der Entnahme im Verhältnis 1:2 mit Perchlorsäure versetzt. Auch Harnproben werden zweckmäßigerweise im gleichen Verhältnis mit Perchlorsäure versetzt. Beim Sammeln des Harns in längeren Perioden wird den Sammelgefäßen Benzoesäure in Substanz zugesetzt.

Der Überstand nach Zentrifugation ist praktisch unbegrenzt haltbar. Harnproben müssen in der Regel noch einmal im Verhältnis 1:10 mit bidest. Wasser verdünnt werden. 


\section{Bestimmungsansatz}

Meßstrahlung: $366(334,340) \mathrm{nm}$; Schichtdicke: $1 \mathrm{~cm}$; Testvolumen: $1,27 \mathrm{ml}$, Kleinküvetten, Raumtemperatur, Messung gegen Luft.

Nacheinander in die Küvette pipettieren:

\begin{tabular}{lccc}
\hline & Leerwert & Probe & $\begin{array}{c}\text { Konzentration } \\
\text { im Test }\end{array}$ \\
\hline $\begin{array}{l}\text { Probe (Überstand nach } \\
\text { Enteiweißung) }\end{array}$ & - & $0,02 \mathrm{ml}$ & \\
$\begin{array}{l}\text { Wasser/Perchlorsäure im } \\
\text { Verhältnis 1:2 }\end{array}$ & $0,02 \mathrm{ml}$ & - & \\
$\begin{array}{l}\text { Tris-Puffer (Lösung 1) } \\
\text { NAD-Lösung (Lösung 2) }\end{array}$ & $1,00 \mathrm{ml}$ & $1,00 \mathrm{ml}$ & $0,79 \mathrm{mmol} / 1$ \\
\hline
\end{tabular}

Gut mischen. $E_{1}$ von Leerwert und Probenansatz messen.

Sorbitdehydrogenase

(Lösung 3) $\quad 0,05 \mathrm{ml} \quad 0,05 \mathrm{ml} \quad 40 \mathrm{mg} / 1$

Nach $30 \min E_{2}$ von Leerwert und Probenansatz messen.

Ist die Reaktion nach 30 min wegen einer hohen Xylit-Konzentration noch nicht zum Stillstand gekommen, so sollte der Ansatz mit einer Probenverdünnung 1:10 wiederholt werden.

$$
\Delta \mathrm{E}^{\perp}=\mathrm{E}_{2}-\mathrm{E}_{1}-\Delta \mathrm{E}_{\text {Leerwert }}
$$

\section{Berechnung}

Die Reaktion läuft unter den angegebenen Bedingungen stöchiometrisch. Der Umrechnungsfaktor berechnet sich aus dem molaren Extinktionskoeffizienten $\varepsilon$ für NADH und der Probenverdünnung durch Vorbehandlung und Bestimmungsansatz. Der Umrechnungsfaktor beträgt für Harnproben, die im Verhältnis 1:10 verdünnt wurden $\mathrm{F}=58500(\mathrm{mg} / \mathrm{l})$ oder $385(\mathrm{mmol} / \mathrm{l})$; für Vollblut, das unverdünnt zur Enteiweißung mit Perchlorsäure versetzt wurde $\mathrm{F}=4970(\mathrm{mg} / \mathrm{l})$ oder $32,7(\mathrm{mmol} / \mathrm{l})$.

\section{Präzision und Richtigkeit}

1. Hamprobe nach mehrstündiger Xylit-Infusion

$$
\begin{aligned}
& (10 \mathrm{~g} / \mathrm{h}), \mathrm{n}=10 ; \\
& \overline{\mathrm{x}}=4867 \mathrm{mg} / \mathrm{l} \\
& \mathrm{s}=187 \mathrm{mg} / \mathrm{l} \\
& \mathrm{VK}=3,8 \%
\end{aligned}
$$

2. Heparin-Blut ohne Xylit-Gehalt + Einwaage von $100 \mathrm{mg} / 100 \mathrm{ml}$ Xylit; $\mathrm{n}=10$;

$\overline{\mathrm{x}}=977 \mathrm{mg} / \mathrm{l}$

$\mathrm{s}=23,9 \mathrm{mg} / \mathrm{l}$

$\mathrm{VK}=2,45 \%$.

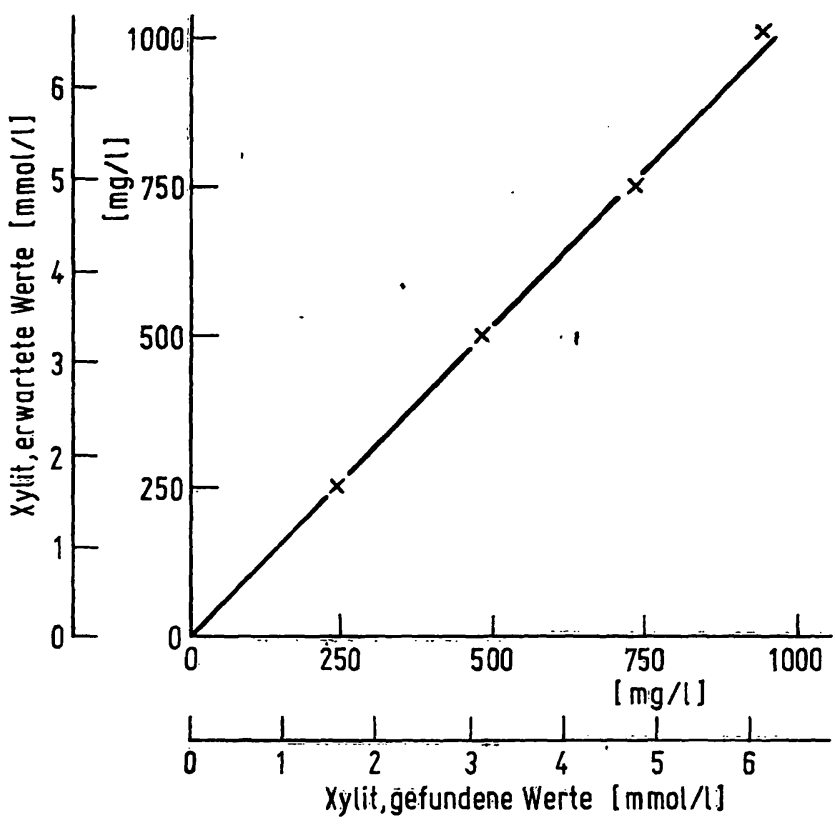

Abb. 1

Korrelation zwischen erwarteten und gefundenen Xylit-Konzentra$(x)=$ jeweils $\bar{x}$ aus $n=5$

3. Korrelation zwischen erwarteten und gefundenen Konzentrationen:

Vollblut 250, 500, 750 und $1000 \mathrm{mg} / \mathrm{l}$

Korrelationskoeffizient $\mathrm{r}=0,997$ s. Abbildung 1 .

4. Um die Störung des Xylit=Nachweises durch Fructose zu beurteilen, wurden je 5 Blutproben mit einem Xylit-Gehalt von $2500 \mathrm{mg} / \mathrm{l}$ mit und ohne Zusatz von $1000 \mathrm{mg} / 1$ Fructose untersucht:

a) Tris-Puffer $1 \mathrm{~mol} / 1 \mathrm{pH} 8,5$ ohne Hydrazin und ohne Fructose-Zusatz. $\quad \overline{\mathrm{x}}=1720 \mathrm{mg} / \mathrm{l}$.

b) Tris-Puffer $1 \mathrm{~mol} / \mathrm{l} \mathrm{pH} 8,5$ ohne Hydrazin + $1000 \mathrm{mg} / 1$ Fructose. $\quad \overline{\mathbf{x}}=1630 \mathrm{mg} / \mathrm{l}$.

c) Tris-Puffer $1 \mathrm{~mol} / \mathrm{l}$ Hydrazin $0,4 \mathrm{~mol} / \mathrm{l}$ ohne Fructose-Zusatz. $\quad \overline{\mathrm{x}}=2370 \mathrm{mgl}$.

d) Tris-Puffer $1 \mathrm{~mol} / \mathrm{l}$ Hydrazin $0,4 \mathrm{~mol} / 1+1000 \mathrm{mg} / \mathrm{l}$ Fructose.

$$
\overline{\mathrm{x}}=2380 \mathrm{mg} / \mathrm{l} \text {. }
$$

\section{Störungen}

Sorbit stört die Nachweisreaktion, doch wird in der parenteralen Ernährung vorwiegend die KohlenhydratKombination Glucose/Fructose/Xylit verwendet, da Sorbit im Organismus ohnehin erst in Fructose umgewandelt wird (2). Die übrigen Polyalkohole wie LIdit, Ribit, Sedoheptit und Allit finden in der parenteralen Ernährung keine Verwendung. Glycerin stört den Nachweis nicht.

\section{Literatur}

1. Bässler, K. H. (1970), in: Methoden der enzymatischen Ana- Verlag Chemie, Weinheim/Bergstr. - 2. BässLer, K. H. (1971), lyse. (Bergmeyer, H. U., Hrsg.) 2. Auflage S. 1344-1347,

Z. Ernährungswissenschaften Suppl. 10, 57-72.

Priv. Doz. Dr. C. Maurer

Klinisch-Chemisches Laboratorium der

Chirurgischen Universitätsklinik Heidelberg Kirschnerstr. 1 